\title{
Microstability of magnetically confined electron-positron plasmas
}

\author{
P. Helander
}

Max-Planck-Institut für Plasmaphysik, 17491 Greifswald, Germany

It is shown that magnetically confined electron-positron plasmas can enjoy remarkable stability properties. Many of the microinstabilities driving turbulence and transport in electron-ion plasmas are absent if the density is so low that the Debye length is significantly larger than the gyroradius. In some magnetic configurations, almost complete linear stability may be attainable in large parts of parameter space. 
Electron-positron plasmas are of great importance in astrophysics and constitute the simplest kind of plasma imaginable, the "hydrogen atom of plasma physics" so to speak. Such an object has not yet been created on Earth, but efforts are underway to do so by accumulating enough positrons from a powerful source and injectng these into a magnetic field, so that a stationary, quasineutral electron-positron laboratory plasma is formed [1]. In such an experiment, a number of important theoretical predictions could be tested experimentally, such as the absence of Faraday rotation, drift waves and sound waves [2]. In the present Letter, we point out that, in addition, such a plasma could enjoy remarkable stability properties quite unlike those of other laboratory plasmas. The common drift-wave and electrostatic interchange instabilities driving turbulence and spoiling confinement in tokamaks and other magnetic-confinement fusion devices should largely be absent, in particular if the magnetic geometry is chosen judiciously.

Positrons can be produced in a number of ways, for example by beta-decay of Na-22 or by pair production from $\mathrm{MeV}$ gamma rays created by nuclear capture of thermal neutrons. These can then be moderated to sub-eV kinetic energies (see eg. [3] or [4]); relatively intense cold positron beams can be extracted [5], and the cold positrons subsequently trapped [6]. In a first laboratory electron-positron plasma, the aim is to produce a (positron $=$ electron) density in the range $10^{12} \mathrm{~m}^{-3}<n<10^{13} \mathrm{~m}^{-3}$ and a temperature $T$ between 1 and $10 \mathrm{eV}$. The Debye length $\lambda_{D}=\left(\epsilon_{0} T / 2 n e^{2}\right)^{1 / 2}$ is then a few $\mathrm{mm}$ and exceeds the gyroradius by two to three orders of magnitude if the magnetic field is about $1 \mathrm{~T}$. The system qualifies as a plasma if its macroscopic dimension $L$ exceeds the Debye length by a large factor. The gyroradius is thus very much smaller than $L$, and any microinstabilities are expected to be well described by conventional gyrokinetic theory. The collision frequency $\nu_{e}$ is much larger than the inverse of the expected confinement time, so the plasma will be in local thermodynamic equilibrium, but at the same time $\nu_{e}$ is smaller than the frequency of typical microinstabilities, so these can be treated as being collisionless.

We use gyrokinetic theory to analyze the stability of such an electron-positron plasma confined by a magnetic field $\mathbf{B}=\nabla \psi \times \nabla \alpha$, where $\psi$ denotes the magnetic flux inside a surface of constant pressure and the coordinate $\alpha$ labels the different field lines on such a magnetic surface. We thus write the distribution function of the two species 
( $a=e$ and $p$, respectively) as

$$
f_{a}=f_{a 0}\left(1-\frac{e_{a} \phi}{T_{a}}\right)+g_{a}
$$

where $f_{a 0}(\psi)$ is Maxwellian, $\phi$ denotes the perturbed electrostatic potential, and $g_{a}$ satisfies the linear gyrokinetic equation in the electrostatic approximation $[7,8]$,

$$
i v_{\|} \nabla_{\|} g_{a}+\left(\omega-\omega_{d a}\right) g_{a}=\frac{e_{a} \phi}{T_{a}} J_{0}\left(\frac{k_{\perp} v_{\perp}}{\Omega_{a}}\right)\left(\omega-\omega_{* a}^{T}\right) f_{a 0} .
$$

Here $\Omega_{a}=e_{a} B / m_{a}$ denotes the gyrofrequency, $\mathbf{k}_{\perp}=k_{\psi} \nabla \psi+k_{\alpha} \nabla \alpha$ the perpendicular wave vector, $\omega_{* a}^{T}=\omega_{* a}\left[1+\eta_{a}\left(m_{a} v^{2} / 2 T-3 / 2\right)\right]$ with $\eta_{a}=d \ln T_{a} / d \ln n_{a}$ and $\omega_{* a}=$ $\left(T_{a} k_{\alpha} / e_{a}\right) d \ln n_{a} / d \psi$ the diamagnetic frequency, and $\omega_{d a}=\mathbf{k}_{\perp} \cdot \mathbf{v}_{d a}$ the magnetic drift frequency. Within factors of order unity (depending on the magnetic geometry) these frequencies are of order $\omega_{d} \sim \omega_{*} \sim\left(k_{\perp} \rho\right) v_{T} / L$, where $v_{T}$ denotes the thermal speed and $\rho$ the gyroradius. The electrostatic potential, finally, is determined by Poisson's law, which for a pure electron-positron plasma becomes

$$
\left(1+k_{\perp}^{2} \lambda_{D}^{2}\right) \phi=\frac{T}{2 n e} \int\left(g_{p}-g_{e}\right) J_{0} d^{3} v
$$

The first notable prediction of these equations is obtained by considering a straight magnetic field by taking $\omega_{d a}=0$ and setting $\nabla_{\|}=i k_{\|}$in equation (1), giving

$$
g_{a}=\frac{\omega-\omega_{* a}^{T}}{\omega-k_{\|} v_{\|}} \frac{e_{a} J_{0} \phi}{T} f_{a 0} .
$$

When this solution is substituted into equation (2), the terms involving $\omega_{* a}^{T}$ cancel and one merely finds a Landau-damped sound wave. Thus, as is well known, there are no drift waves in a pure electron-positron plasma. Perhaps more surprisingly, there is no possiblity of instability: what in an electron-ion plasma are known as the slab branches of the ion- and electron-temperature-gradient modes are stable [9]. In other words, any instability must involve magnetic curvature.

In order to analyse curvature-driven modes, we first take the high-frequency limit, $k_{\|} v_{T} \ll \omega$, in equation (1). The solution becomes

$$
g_{a}=\frac{\omega-\omega_{* a}^{T}}{\omega-\omega_{d a}} \frac{e_{a} J_{0} \phi}{T} f_{a 0},
$$

and the dispersion relation from equation (2)

$$
1-I_{0}(b) e^{-b}+k_{\perp}^{2} \lambda_{D}^{2}=\frac{1}{n} \int \frac{\omega_{d}\left(\omega_{d}-\omega_{*}^{T}\right)}{\omega^{2}-\omega_{d}^{2}} J_{0}^{2} f_{0} d^{3} v,
$$


where $b=k_{\perp}^{2} T / m \Omega^{2}$ and $I_{0}$ denotes a modified Bessel function. Here and henceforth, a subscript $a=p$ is understood where appropriate. Because $\omega_{d}$ and $\omega_{*}$ are comparable, the right-hand-side of this equation is at most of order unity, and any instability must therefore have a perpendicular wavelength comparable to the Debye length, $k_{\perp} \lambda_{D}=$ $O(1)$. Since this would contradict the assumption $k_{\|} v_{T} \ll \omega$ when $\rho \ll \lambda_{D}$, we conclude that no high-frequency modes are possible.

We are thus led to the opposite limit, $\omega \ll k_{\|} v_{T}$, in which the solution of equation (1) instead becomes

$$
g_{a}=\frac{\omega-\omega_{* a}^{T}}{\omega-\bar{\omega}_{d a}} \frac{e_{a} \bar{\phi}}{T} f_{a o}
$$

where an overhead bar denotes an orbit average. Substituting this solution into Poisson's law (2) now gives an integral equation for the mode structure,

$$
\left(1+k_{\perp}^{2} \lambda_{D}^{2}\right) \phi=\frac{1}{n} \int \bar{\phi}\left(1+\frac{\bar{\omega}_{d}\left(\bar{\omega}_{d}-\omega_{*}^{T}\right)}{\omega^{2}-\bar{\omega}_{d}^{2}}\right) f_{0} d^{3} v
$$

which again implies $k_{\perp} \lambda_{D} \lesssim O(1)$. Whatever magnetic geometry is used, we thus expect that instabilities with $k_{\perp} \rho=O(1)$ do not exist. This is in stark contrast to electronion plasmas, where such instabilities are dominant and drive the observed turbulent transport.

Any remaining longer-wavelength-instabilities predicted by the eigenvalue problem (3) would however cause turbulence and transport, which could be similar to that in conventional plasmas except that the wavelength is of order $\lambda_{D}$ instead of $\rho$. There is, however, an important difference. Since $\omega \ll k_{\|} v_{T}$, the action integral for trapped orbits,

$$
J=\int m v_{\|} d l
$$

is conserved. This integral is taken along the magnetic field (the arc length is denoted by $l$ ) between points where $v_{\|}$vanishes, and is adiabatically invariant under slow perturbations. The derivatives of $J$ are related to the orbit-averaged drift velocity [10, 11],

$$
\begin{aligned}
& \overline{\mathbf{v}_{d} \cdot \nabla \psi}=\frac{1}{e_{a} \tau_{b}} \frac{\partial J}{\partial \alpha}, \\
& \overline{\mathbf{v}_{d} \cdot \nabla \alpha}=-\frac{1}{e_{a} \tau_{b}} \frac{\partial J}{\partial \psi},
\end{aligned}
$$

where

$$
\tau_{b}=\int \frac{d l}{v_{\|}}=\int \frac{d l}{v \sqrt{1-\lambda B}}
$$


denotes the bounce time in magnetic trapping wells between two points of equal magnetic field strength and $\lambda=v_{\perp}^{2} /\left(v^{2} B\right)$. These wells are defined by the condition $\lambda B<1$, and the integration along the field is carried out between points where $\lambda B=1$. A perfectly confining, so-called omnigenous, magnetic field is one where the average drift velocity has no component in the $\psi$-direction [12]. Such magnetic configurations, where thus $\partial J / \partial \alpha=0$, are sought in stellarator and magnetic-mirror research, and can be found to a good approximation, but not exactly [13].

Already in 1968, Rosenbluth [14] suggested that magnetic configurations should be stable to low-frequency interchange modes if $J$ decreases away from the centre of the plasma. His argument for the stability in such so called maximum- $J$ configurations was recently extended and applied to stellarators $[15,16]$. In the present context, it can be obtained by multiplying equation (3) by $\phi^{*} / B$ and integrating over velocity space and along the magnetic field. This gives the quadratic form

$$
\begin{gathered}
\int\left(1+k_{\perp}^{2} \lambda_{D}^{2}\right)|\phi|^{2} \frac{d l}{B}-\frac{1}{2} \int \sum_{j}\left|\bar{\phi}_{j}\right|^{2} \tau_{b j} d \lambda \\
=\frac{1}{n} \int_{0}^{\infty} f_{0} 2 \pi v^{3} d v \int \sum_{j}\left|\bar{\phi}_{j}\right|^{2} \tau_{b j} \frac{\bar{\omega}_{d}\left(\bar{\omega}_{d}-\omega_{*}^{T}\right)}{\omega^{2}-\bar{\omega}_{d}^{2}} d \lambda,
\end{gathered}
$$

where the sum over $j$ is taken over all magnetic wells along the field line, and $\tau_{b j}$ denotes the bounce time in the $j$ :th well. The imaginary part of this equation implies that $\omega^{2}$ is real, so that the eigenmodes are either purely oscillatory or exponentially growing/damped. It thus follows from the real part of equation (4) that, if an instability exists, then the product $\omega_{*}^{T} \bar{\omega}_{d}$ cannot be negative everywhere. However, this product is equal to

$$
\omega_{*}^{T} \bar{\omega}_{d}=-\frac{k_{\alpha}^{2} T}{e^{2} \tau_{b j}} \frac{d \ln n}{d \psi}\left(\frac{\partial J}{\partial \psi}-\frac{k_{\psi}}{k_{\alpha}} \frac{\partial J}{\partial \alpha}\right)\left[1+\eta\left(\frac{m v^{2}}{2 T}-\frac{3}{2}\right)\right],
$$

where in a well-optimized magnetic configuration $\partial J / \partial \alpha$ is small in comparison with $\partial J / \partial \psi$. In an omnigenous device, $\partial J / \partial \alpha=0$ and the product $\omega_{*}^{T} \bar{\omega}_{d}$ is negative for all orbits if the magnetic field satisfies the maximum- $J$ condition, $0<\eta<2 / 3$, and the density peaks in the centre of the plasma. The right-hand side of equation (4) can then only be positive if $\omega^{2}>0$, and we arrive at the remarkable conclusion that there is no instability, regardless of how large the density gradient is made (within the orderings assumed). Thus, whereas in electon-ion plasmas the maximum- $J$ condition 
merely suppresses certain types of instabilities (the low-frequency end of the spectrum), it apparently leads to complete stability against electrostatic modes in an electronpositron plasma with $\rho \ll \lambda_{D}$ and $0<\eta<2 / 3$, if the magnetic field is perfectly omnigenous. If it is only approximately so, the only instabilities possible are those with very large radial mode numbers, $k_{\psi} \partial J / \partial \alpha>k_{\alpha} \partial J / \partial \psi$. At least according to quasilinear theory, such instabilities should not lead to large transport.

The simplest electron-positron experiment to build would not be a maximum- $J$ configuration but something less complicated. The very simplest alternative is perhaps a magnetic dipole field, created either by a mechanically suspended permanent magnet or by a levitated circular coil. A plasma confined by a dipole field has the peculiar property of being stable to low-frequency modes if the equilibrium distribution function only depends on the adiabatic invariants $\mu$ and $J$ but not on the flux-surface label $\psi[17]$. However, such a distribution function cannot be Maxwellian and therefore does not apply to a plasma where the collision frequency exceeds the inverse confinement time. If $f_{0}$ is Maxwellian, the threshold for instabilities with $\omega \ll k_{\|} v_{T}$ satisfies equation (4) with $\omega=0$, and can be obtained to a good approximation by taking $\omega_{d}$ to be independent of $\lambda[18]$. In this approximation, the eigenfunction $\phi$ becomes constant along the magnetic field and the stability boundary for electrostatic modes becomes

$$
\frac{d \ln n}{d \ln U}=\frac{1}{3(\eta-1)}
$$

where $U(\psi)=V^{\prime}(\psi)$ and $V(\psi)$ denotes the volume enclosed by the magnetic surface labelled by $\psi$. For a dipole field, $\psi$ is proportional to $\sin ^{2} \theta / r$ in spherical coordinates, $V^{\prime}(\psi) \propto \psi^{-4}$, and the stability criterion can be written as

$$
\frac{d \ln (n / T)}{d \ln \psi}<\frac{4}{3}
$$

The calculation leading to this result is similar to that in a conventional ion-electron plasma considered in Ref. [18] and is therefore not repeated here, but we note that the result is much stronger in the present context. Because the Debye length is much larger than the gyroradius, high-frequency modes ( $\omega$ of order $k_{\|} v_{T}$ or larger) are guaranteed to be stable and there is no need to make an explicit assumption about low frequency. It is interesting to note that the temperature gradient is stabilizing for these modes.

The achievable plasma pressure can also be limited by electromagnetic instabilities, which should behave very differently in the case of a maximum- $J$ device and a 
magnetic dipole. The former tend to have a magnetic well [19] and are therefore stable against magnetohydrodynamic (MHD) modes up to a finite normalized pressure $\beta=2 \mu_{0} p / B^{2}$, where $p=2 n T$. Since $\beta$ is exceedingly small for the electron-positron plasma parameters mentioned above, unstable MHD interchanges or ballooning modes are not expected. In a dipole, however, the magnetic curvature is unfavourable, and such modes are unstable if

$$
\frac{d\left(p U^{5 / 3}\right)}{d \ln \psi}>0 \Rightarrow \frac{d \ln (n T)}{d \ln \psi}>\frac{20}{3} .
$$

An arbitrarily small pressure gradient can thus be MHD unstable if the pressure itself is small enough. On the other hand, the marginally stable pressure profile is very steep, as emphasized by Hasegawa, Chen and Mauel [17]. The stability diagram corresponding to equations (5) and (6) is shown in Fig. 1.

In summary, it has been found that the electrostatic instabilities causing turbulence and transport in magnetically confined electron-ion plasmas are largely absent in low-density electron-positron plasmas. Only low-frequency interchange modes can be unstable, and in some magnetic configurations almost complete linear stability may be attainable. It should be emphasized that two requirements are essential for these conclusions: the absence of ions removes the slab ion- and electron-temperature-gradient modes, and the very low density expected in electron-positron plasmas causes the Debye length to be large enough to stabilize short-wavelength modes. Neither of these conditions is satisfied in fusion experiments.

\section{Acknowledgement}

The author is grateful to T. Sunn Pedersen and J.W. Connor for valuable comments and helpful discussions. 


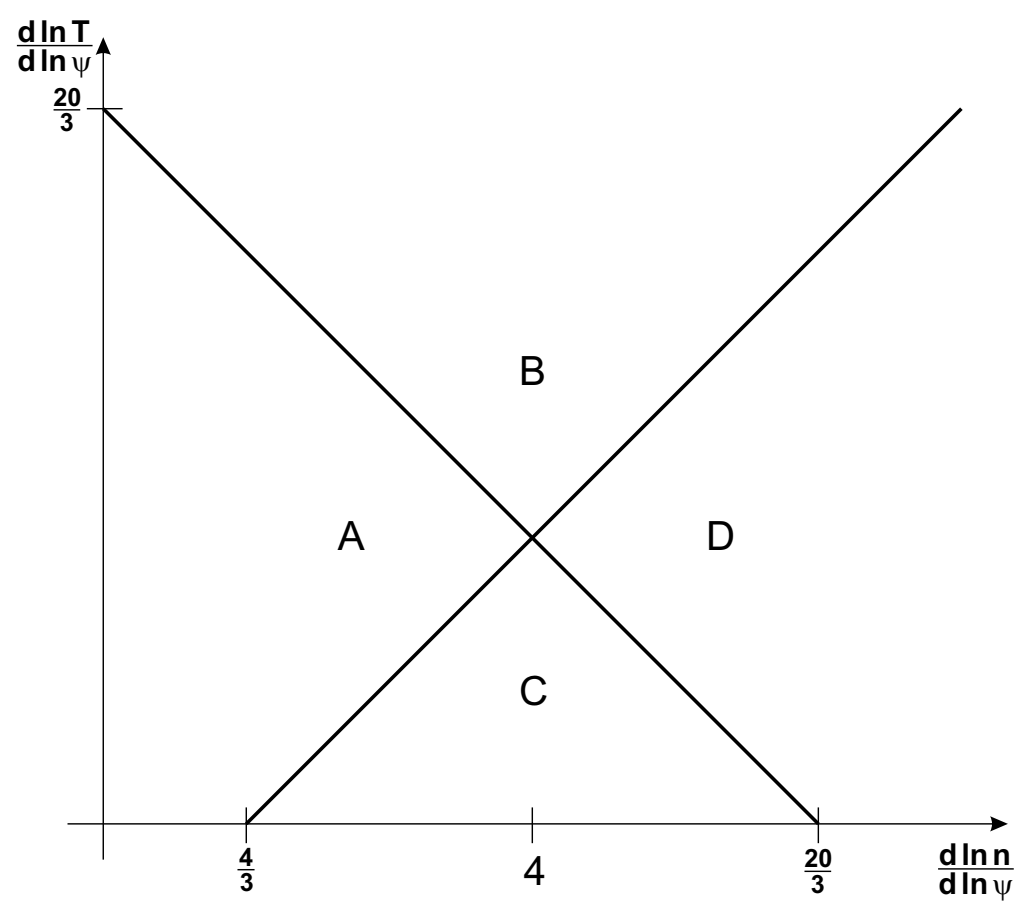

Figure 1: Stability diagram of an electron-positron plasma in a dipole magnetic field. Regions $A$ and $B$ are stable to electrostatic modes, whilst regions $B$ and $D$ are unstable to MHD interchanges.

\section{References}

[1] T. Sunn Pedersen, J.R. Danielson, C. Hugenschmidt, G. Marx, X. Sarasola, F. Schauer, L. Schweikhard, C.M. Surko and E. Winkler, New J. Phys. 14, 035010 (2012).

[2] V. Tsytovich and C.B. Wharton, Comments Plasma Phys. Contr. Fusion 4, 91 (1978).

[3] R.G. Greaves and C.M. Surko, Canad. J. Phys. 74, 445 (1996).

[4] C. Hugenschmidt, G. Kögel, R. Repper, K. Schreckenbach, P. Sperr and W. Triftshäuser, Nuclear Instruments and Methods in Physics Research Section B: Beam Interactions with Materials and Atoms 198, 220 (2002).

[5] C. Hugenschmidt, K. Schreckenbach, M. Stadlbauer and B. Straver, Nuclear Instruments and Methods in Physics Research Section A: Accelerators, Spectrometers, Detectors and Associated Equipment 554, 384 (2005). 
[6] C.M. Surko and T.J. Murphy, Phys. Rev. A 46, 5696 (1992).

[7] P.H. Rutherford and E.A. Frieman, Phys. Fluids 11, 569 (1968).

[8] J.B. Taylor and R.J. Hastie, Plasma Phys. 10, 479 (1968).

[9] T. Sunn Pedersen, A.H. Boozer, W. Dorland, J.P. Kremer and R. Schmitt, J. Phys. B: At. Mol. Opt. Phys. 361029 (2003).

[10] B.B. Kadomtsev and O.P. Pogutse, Nucl. Fusion 11, 67 (1971).

[11] M.N. Rosenbluth and M.L. Sloan, Phys. Fluids 14, 1725 (1971).

[12] L.S. Hall and B. McNamara, Phys. Fluids 18, 552 (1975).

[13] J.R. Cary and S. Shasharina, Phys. Plasmas 4, 3323 (1997).

[14] M.N. Rosenbluth, Phys. Fluids 11, 869 (1968).

[15] J.H.E. Proll, P. Helander, J.W. Connor and G.G. Plunk, Phys. Rev. Lett. 108, 245002 (2012); P. Helander, J.H.E. Proll and G.G. Plunk, Phys. Plasmas 20, 122505 (2013).

[16] P. Helander, C.D. Beidler, T.M. Bird, M. Drevlak, Y. Feng, R. Hatzky, F. Jenko, R. Kleiber, J.H.E. Proll, Yu. Turkin and P. Xanthopoulos, Plasma, Phys. Control. Fusion 54, 124009 (2012).

[17] A. Hasegawa, L. Chen and M.E. Mauel, Nucl. Fusion 30, 2405 (1990).

[18] J. Kesner and R.J. Hastie, Phys. Plasmas 9, 395 (2002).

[19] P. Helander, Rep. Prog. Phys. 77, 087001 (2014). 